\title{
Constrained power allocation schemes for coordinated base station transmission using block diagonalization
}

\author{
Ana García Armada1*, Matilde Sánchez-Fernández ${ }^{1}$ and Roberto Corvaja²
}

\begin{abstract}
In this study, we propose several power allocation schemes in a coordinated base station downlink transmission with per antenna and per base station power constraints. Block Diagonalization is employed to remove interference among users. For each set of power constraints, two schemes based on the waterfilling distribution are proposed and compared to the optimal solution, which can only be obtained numerically by using convex optimization. We show that the proposed schemes achieve a performance, in terms of weighted sum rate, very close to the optimal, without the heavy computational complexity required by the latter. The sum rates are compared first in a simplified two-user two-cell case where we also compare our approach to the previous solutions available in the literature. Then, we examine the performance in a multi-cell scenario where we also evaluate the degradation of the performance caused by imperfect channel state information.
\end{abstract}

Keywords: coordinated base stations, multiple-antennas, network MIMO, block diagonalization

\section{Introduction}

Space-division multiplexing (SDM) based on multiple input-multiple output (MIMO) techniques emerged as a means of achieving high-capacity communications [1]. However, the introduction of MIMO processing in cellular networks does not offer the expected benefits, the main reason being the interference that characterizes these environments. SDM requires high signal-to-noise-plusinterference ratios (SINR) to leverage its capacity-achieving potential. Unfortunately, the interference in cellular systems lowers the operating point toward low SINR, thus making MIMO processing not so advantageous. Recently some study has been devoted to manage interference in cellular systems with reuse one, where all cells are allowed to use the same frequencies, also known as universal frequency reuse [2]. In [3], the other-cell interference (OCI) is considered when designing the transmission for a multiuser MIMO downlink. In [4], the authors analyze several approaches for overcoming interference in SDM MIMO cellular networks. If the interference is known by the

\footnotetext{
* Correspondence: agarcia@tsc.uc3m.es

'Department of Signal Theory and Communications, Universidad Carlos III de Madrid, Leganés, Spain

Full list of author information is available at the end of the article
}

transmitters, then cooperative encoding among base stations using dirty paper coding (DPC) can suppress OCI [5]. In [6], several strategies are proposed to perform coordinated base station transmission (CBST). Interference is eliminated by jointly and coherently coordinating the transmission from the base stations in the network, assuming that base stations know all downlink signals. Besides DPC, they propose a zero-forcing (ZF) scheme that, although suboptimal, does not involve the complexity of DPC. The capacity of MIMO benefits from CBST not only because of the rise of the operating SINR point, but also from the better rank condition of the joint channel matrix resulting from non-collocated base stations [7].

Similar to multi-user MIMO, block diagonalization (BD) $[8,9]$ may be applied for CBST as a good compromise between complexity and performance. In [10], BD is applied in a multicell scenario in combination with the OCI reduction scheme of [3]. Alternatively, in [11], a singular value decomposition (SVD) approach is proposed that simplifies the channel estimation requirements at the expense of a performance degradation.

In this article, we focus on BD-based CBST with different power constraints at the transmission side, with the aim of maximizing the weighted sum rate (WSR) of the 
users in a cellular network. A first reasonable assumption for power constraints is to consider that each base station (BS) has restricted its total transmission power; this was used, for example, in $[6,10,12]$. Alternatively, per antenna constraints may be more realistic, since each transmission antenna is usually driven by its own high-power amplifier [13].

In this article, we consider both per base station and per antenna restrictions. For each of them, we will formulate the optimization problem and derive two power allocation schemes that resemble the well-known waterfilling (WF) distribution. While WF is known to achieve capacity in single-user frequency-selective transmission [14], modified versions of WF also give the capacity-achieving power allocation in multi-user communications $[15,16]$. In [10], a scaled WF (SWF) scheme is heuristically proposed for the case of per base station power constraints to avoid a lengthy numerical optimization. However, its performance is not discussed nor compared to optimal approaches. In [7], a BD scheme denoted as JT-decomp is proposed where the powers are assigned to the users' transmissions with the only aim of insuring that per base station power constraints are fulfilled. No optimization is performed on the transmit powers to maximize the achievable rates. Consequently, the obtained rates are lower. Also, some partial results of the study shown here, again only for the case of per base station power constraints, have been presented in [17]. We will show that the schemes that we are proposing, although suboptimal, perform very close to the optimum power allocation-obtained by numerical convex optimization-with a reduced complexity.

In brief, the innovative contributions of this article are the following. We develop closed-form and implementable solutions for the power allocation in a BD-based CBST system with realistic power constraints at the transmission side. These solutions are not empirical, but they are obtained, starting from the optimal allocation, using only few approximations that allow us to understand why they perform close to the optimum. In the case of per base station power constraints, one of our proposals gives the same result as SWF [10], while the others are new. We show also that our approaches reduce dramatically the complexity with respect to the optimal search. Moreover, we consider also the effect of errors in the channel estimation and of a time-varying channel, in which the use of outdated channel state information due to the feed-back delay reduces the achievable rates.

The remainder of this article is structured as follows. In the next section, the system model is presented; in the "Constrained optimization and optimal power allocation" section, the optimization problem is described; while in the "Waterfilling distributions for suboptimal power allocation schemes" section the proposed power allocation schemes with per base station and per antenna constraints are developed. The "Numerical results" section discusses some performance results and the "Complexity" section explores the complexity of the proposed solutions. The article concludes with some concluding remarks.

Notations: In this article, the following notations will be used. Boldface symbols will be used for matrices and vectors, while italic letters will be used for scalars. Superscripts ${ }^{T}$ and ${ }^{H}$ denote the transpose and the Hermitian transpose of a matrix, respectively; superscript * refers to an optimal solution; $[\cdot]^{+}$denotes the maximum between zero and the argument; and $\|\cdot\| \|_{F}$ denotes de Frobenius norm of a matrix.

\section{System model}

The system model assumes a coordinated transmission downlink scenario, where $M$ base stations serve $N$ users. Each base station has $t$ transmit antennas, and each user has $r$ receive antennas. Although our analysis is general, the performance will be illustrated for BS-user pairs; therefore the case $M=N$ will be considered in the "Results" section.

Assuming narrowband transmission (if the channel is frequency selective, it can be decomposed into a number of parallel non-interfering subchannels, each experiencing approximately frequency-flat fading), the channel may be modeled by a $\mathrm{Nr} \times \mathrm{Mt}$ channel matrix $\mathbf{H}$ where each matrix coefficient represents the gain from each transmit antenna in the BS to each receive antenna at the user side.

The received signal model is as follows:

$$
\mathbf{y}=\mathbf{H x}+\mathbf{n}
$$

where $\mathbf{y}$ is the received $N r \times 1$ signal vector, $\mathbf{x}$ is the $M t \times 1$ signal vector transmitted from all the BSs, and $\mathbf{n}$ is a $N r \times 1$ vector of i.i.d complex Gaussian entries with zero mean and unit variance. If we define $\mathbf{H}_{i}$ with $i=$ $1 \ldots N$ as the $r \times M t$ channel matrix seen by user $i$, then $\mathbf{H}=\left[\mathbf{H}_{1}^{T} \mathbf{H}_{2}^{T} \ldots \mathbf{H}_{N}^{T}\right]^{T}$. Figure 1 illustrates the reference scenario for the particular case of $M=N=3, t=3$ and $r=1$.

For the CBST scenario, we define $\mathbf{x}$ as follows:

$$
\mathbf{x}=\sum_{j=1}^{r} b_{1 j} \mathbf{w}_{1 j}+\sum_{j=1}^{r} b_{2 j} \mathbf{w}_{2 j}+\cdots+\sum_{j=1}^{r} b_{N j} \mathbf{w}_{N j}=\mathbf{W b}
$$

where $\mathbf{b}=\left[b_{11}, \ldots, b_{1 r}, \ldots, b_{N r}\right]^{T}, b_{i j}$ represents the $j$ th symbol for user $i$ transmitted with power $P_{i j}$, the precoding matrix is defined as $\mathbf{W}=\left[\mathbf{w}_{11}, \ldots, \mathbf{w}_{1 r}, \ldots, \mathbf{w}_{N r}\right]$ and $\mathbf{w}_{i j}=\left[w_{i j}^{11}, \ldots, w_{i j}^{1 t}, \ldots, w_{i j}^{k l}, \ldots, w_{i j}^{M t}\right]^{T}$ are the precoding vectors. 


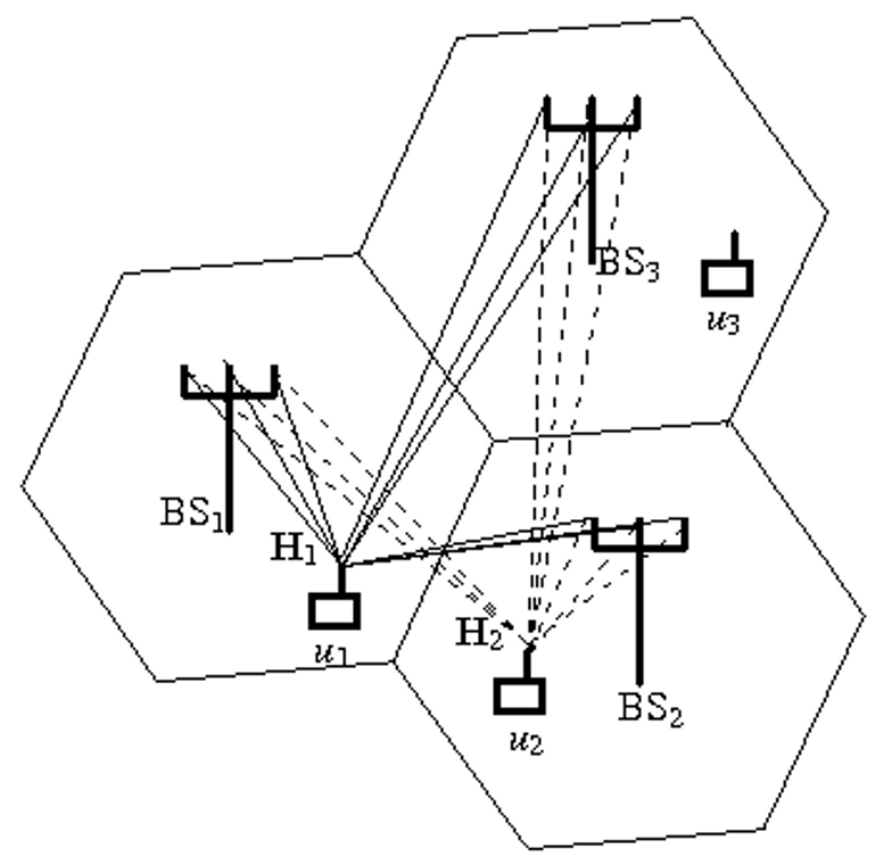

Figure 1 Illustration of the system model for $M=3$ base stations each equipped with $t=3$ transmit antennas, $N=3$ users, each with $r=1$ receive antenna. The solid lines are used for channel $H_{1}$ experienced by user 1 , and the dotted lines $\mathrm{H}_{2}$ for user 2 .

The precoding sub-matrices $\mathbf{W}_{i}=\left[\mathbf{w}_{i 1}, \ldots, \mathbf{w}_{i r}\right]$ will be obtained through $\mathrm{BD}$ as in $[6,8,10]$, to guarantee that there is no inter-user interference, that is

$$
\mathbf{H}_{i} \mathbf{W}_{n}=\mathbf{0} \text { if } i \neq n,
$$

where $\mathbf{0}$ is an all-zero matrix of dimensions $r \times r$. If we define $\widetilde{\mathbf{H}}_{i}=\left[\mathbf{H}_{1}^{T} \mathbf{H}_{2}^{T} \ldots \mathbf{H}_{i-1}^{T} \mathbf{H}_{i+1}^{T} \ldots \mathbf{H}_{N}^{T}\right]^{T}$, then the condition (3) is obtained if $\mathbf{w}_{i j}$ lie in the null space of $\widetilde{\mathbf{H}}_{i}$. Let $\widetilde{l}_{i}=\operatorname{rank}\left(\widetilde{\mathbf{H}}_{i}\right)$ and define the SVD

$$
\widetilde{\mathbf{H}}_{i}=\widetilde{\mathbf{U}}_{i} \widetilde{\mathbf{S}}_{i}\left[\widetilde{\mathbf{V}}_{i}^{(1)} \widetilde{\mathbf{V}}_{i}^{(0)}\right]^{H}
$$

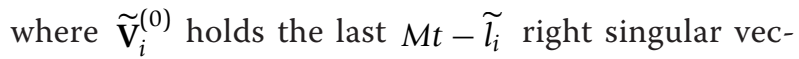
tors. We consider another SVD:

$$
\mathbf{H}_{i} \widetilde{\mathbf{V}}_{i}^{(0)}=\mathbf{U}_{i}\left[\begin{array}{cc}
\mathbf{S}_{i} & \mathbf{0} \\
\mathbf{0} & \mathbf{0}
\end{array}\right]\left[\mathbf{V}_{i}^{(1)} \mathbf{V}_{i}^{(0)}\right]^{H}
$$

where $\mathbf{V}_{i}^{(1)}$ represents the first $l_{i}$ singular vectors, $l_{i}=r$ being the rank of $\mathbf{H}_{i} \widetilde{\mathbf{V}}_{i}^{(0)}$. The product $\mathbf{W}_{i}=\widetilde{\mathbf{V}}_{i}^{(0)} \mathbf{V}_{i}^{(1)}$ represents the transmission vectors that maximize the information rate for user $i$ subject to the condition of canceling interference. Therefore,

$$
\mathbf{H}_{i} \mathbf{W}_{n}=\mathbf{U}_{i} \mathbf{S}_{i} \text { if } i=n
$$

$\mathbf{U}_{i}$ is an $r \times r$ unitary matrix and

$$
\mathbf{S}_{i}=\operatorname{diag}\left\{\lambda_{i 1}^{1 / 2}, \lambda_{i 2}^{1 / 2}, \ldots, \lambda_{i r}^{1 / 2}\right\}
$$

$\lambda_{i j}^{1 / 2}$ being the singular values of $\mathbf{H}_{i} \widetilde{\mathbf{V}}_{i}^{(0)}$. Then, the received signal can be expressed as

$$
\mathbf{y}=\left[\begin{array}{llll}
\mathbf{U}_{1} \mathbf{S}_{1} & \mathbf{0} & \cdots & \mathbf{0} \\
\mathbf{0} & \mathbf{U}_{2} \mathbf{S}_{2} & \cdots & \mathbf{0} \\
\vdots & \vdots & \ddots & \vdots \\
\mathbf{0} & \mathbf{0} & \cdots & \mathbf{U}_{N} \mathbf{S}_{N}
\end{array}\right] \mathbf{b}+\mathbf{n} .
$$

Each user independently rotates the received signal and decouples the different streams:

$$
\widetilde{\mathbf{y}}=\left[\begin{array}{cccc}
\mathbf{U}_{1}^{H} & \mathbf{0} & \cdots & \mathbf{0} \\
\mathbf{0} & \mathbf{U}_{2}^{H} & \cdots & \mathbf{0} \\
\vdots & \vdots & \ddots & \vdots \\
\mathbf{0} & \mathbf{0} & \cdots & \mathbf{U}_{N}^{H}
\end{array}\right] \mathbf{y}=\left[\begin{array}{c}
\lambda_{11}^{1 / 2} b_{11} \\
\vdots \\
\lambda_{1 r}^{1 / 2} b_{1 r} \\
\vdots \\
\lambda_{N r}^{1 / 2} b_{N r}
\end{array}\right]+\widetilde{\mathbf{n}}
$$

where the noise $\tilde{\mathbf{n}}$ remains white with the same covariance because of the unitary transformation. BD is 
possible in this scenario if the condition $M t \geq N r$ is satisfied $[6,8,10]$.

\section{Constrained optimization and optimal power allocation}

Under the BD-CBST strategy, it can be observed from (9) that the overall system is then turned into a set of parallel non-interfering channels. Therefore, the achievable rate of user $i$ is $R_{i}=\sum_{j=1}^{r} \log _{2}\left(1+\lambda_{i j} P_{i j}\right)$.

We would like to maximize a weighted sum of the rates $R_{i}$ for the set of users, which requires solving the following optimization problem in terms of the power $P_{i j}$ allocated to the $j$ th stream of user $i$ :

$$
\max \left\{\sum_{i=1}^{N} \alpha_{i} \sum_{j=1}^{r} \log _{2}\left(1+\lambda_{i j} P_{i j}\right)\right\}
$$

where the values $\alpha_{i} \in[0,1]\left(\sum_{i=1}^{N} \alpha_{i}=1\right)$, can be seen as indicating the priorities of the users: the closer $\alpha_{i}$ is to 1 , the higher the priority given to user $i$. In the particular case of $\alpha_{i}=1 / N$, for all $i$, the solution of the above problem maximizes the sum rate.

In this context, two different constraints on the power available at the transmitter side may be considered. The first one deals with per base station restrictions, where each base station $k$ has a maximum available power $P_{\operatorname{maxBS}}$ to transmit. ${ }^{\text {a }}$ Then, the power allocation $P_{i j}$ should fulfill the following constraints:

$$
\sum_{l=1}^{t} \sum_{i=1}^{N} \sum_{j=1}^{r} P_{i j}\left|w_{i j}^{k l}\right|^{2} \leq P_{\operatorname{maxBS}}
$$

for each BS $k=1, \ldots, M$.

The second set of constraints that may be considered on the power available at the transmitters is given by a restriction of the maximum power $P_{\operatorname{maxAn}}$ transmitted by each antenna of each BS (per antenna constraints). ${ }^{\mathrm{b}}$ The restriction of the maximum power transmitted by each antenna $l=1, \ldots, t$ of each BS $k=1, \ldots, M$, conditions the power allocation $P_{i j}$ as follows:

$$
\sum_{i=1}^{N} \sum_{j=1}^{r} P_{i j}\left|w_{i j}^{k l}\right|^{2} \leq P_{\operatorname{maxAn}}
$$

Maximizing a weighted sum of the rates $R_{i}$ under any of the two proposed constraints is a convex problem, since the logarithmic function is concave in the power assignments: the additional operation preserves concavity, and the constraints (11) are linear. Therefore, the optimal solution may eventually be derived by numerical convex optimization techniques $[18,19]$. However, closed-form solutions, even if suboptimal, are highly preferable, to reduce the computational time and resources required by the CBST for the power allocation. Thus, we approach a closed-form solution of the problem by applying the Lagrange duality theory.

In order to solve the problem (10) with constraints (11) or (12), we can introduce a Lagrangian $\Lambda(\mathbf{P}, \boldsymbol{\mu})$ where $\mathbf{P}$ is the vector collecting all the powers $P_{i j}, i=$ $1, \ldots, N, j=1, \ldots, r$, and $\boldsymbol{\mu}$ contains the (non-negative) Lagrange multipliers.

When we have the per base station constraints (11), $\Lambda(\mathbf{P}, \boldsymbol{\mu})$ is given by

$$
\Lambda(\mathbf{P}, \mu)=\sum_{i=1}^{N} \alpha_{i} \sum_{j=1}^{r} \log _{2}\left(1+\lambda_{i j} P_{i j}\right)-\mu_{1}\left[g_{1}(\mathbf{P})-P_{\max B S}\right]-\cdots-\mu_{M}\left[g_{M}(\mathbf{P})-P_{\operatorname{maxBS}}\right]
$$

with

$$
g_{k}(\mathbf{P})=\sum_{l=1}^{t} \sum_{i=1}^{N} \sum_{j=1}^{r} P_{i j}\left|w_{i j}^{k l}\right|^{2} \quad k=1, \ldots, M
$$

and $\boldsymbol{\mu}=\left[\mu_{1}, \ldots, \mu_{M}\right]$.

Similarly, when the per antenna constraints (12) are applied, $\Lambda(\mathbf{P}, \boldsymbol{\mu})$ is given by

$$
\Lambda(\mathbf{P}, \boldsymbol{\mu})=\sum_{i=1}^{N} \alpha_{i} \sum_{j=1}^{r} \log _{2}\left(1+\lambda_{i j} P_{i j}\right)-\mu_{11}\left[g_{1}^{1}(\mathbf{P})-P_{\max A n}\right]-\cdots-\mu_{M t}\left[g_{M}^{t}(\mathbf{P})-P_{\max A n}\right]
$$

with

$$
g_{k}^{l}(\mathbf{P})=\sum_{i=1}^{N} \sum_{j=1}^{r} P_{i j}\left|w_{i j}^{k l}\right|^{2} \quad k=1, \ldots, M \quad l=1, \ldots
$$

and

$$
\boldsymbol{\mu}=\left[\begin{array}{ccc}
\mu_{11} & \cdots & \mu_{1 t} \\
\vdots & \ddots & \vdots \\
\mu_{M 1} & \cdots & \mu_{M t}
\end{array}\right]
$$

With the per base station constraints, the solution of the problem is given by a point $\left[\mathbf{P}^{*}, \boldsymbol{\mu}^{*}\right]$ that satisfies the set of $\mathrm{Nr}+\mathrm{M}$ equations:

$$
\begin{aligned}
& \frac{\alpha_{i} \lambda_{i j}}{\ln (2)\left(1+\lambda_{i j} P_{i j}\right)}-L_{i j}^{\mathrm{BS}}=0 \quad i=1, \ldots, N \quad j=1, \ldots, r \\
& \sum_{l=1}^{t} \sum_{i=1}^{N} \sum_{j=1}^{r} P_{i j}\left|w_{i j}^{k l}\right|^{2} \leq P_{\max \mathrm{BS}} \quad k=1, \ldots, M
\end{aligned}
$$

with $L_{i j}^{\mathrm{BS}}=\sum_{k=1}^{M} \sum_{l=1}^{t} \mu_{k}\left|w_{i j}^{k l}\right|^{2}$.

Again and similarly, with the per antenna constraints, the solution should satisfy the set of $N r+M t$ equations:

$$
\begin{aligned}
& \frac{\alpha_{i} \lambda_{i j}}{\ln (2)\left(1+\lambda_{i j} P_{i j}\right)}-L_{i j}^{\mathrm{An}}=0 \quad i=1, \ldots, N \quad j=1, \ldots, r \\
& \sum_{i=1}^{N} \sum_{j=1}^{r} P_{i j}\left|w_{i j}^{k l}\right|^{2} \leq P_{\operatorname{maxAn}} \quad k=1, \ldots, M \quad l=1, \ldots, t
\end{aligned}
$$


with $L_{i j}^{\mathrm{An}}=\sum_{k=1}^{M} \sum_{l=1}^{t} \mu_{k l}\left|w_{i j}^{k l}\right|^{2}$

The solution of (18) and (19) is given by the values of $\mathbf{P}^{*}$ and $\boldsymbol{\mu}^{*}$ such that

$$
P_{i j}+\frac{1}{\lambda_{i j}}=\frac{\alpha_{i}}{\ln (2) L_{i j}^{\mathrm{BS} / \mathrm{An}}}
$$

where the vector of Lagrange multipliers $\boldsymbol{\mu}^{*}$, which defines $L_{i j}^{\mathrm{BS} / \mathrm{An}}$, should be chosen so that each set of power constraints is satisfied. It can be observed that in both cases, the solution resembles the well-known WF distribution. However, here the waterlevel is given by $\alpha_{i} /\left(\ln (2) L_{i j}^{\mathrm{BS} / \mathrm{An}}\right)$, that is, the waterlevel is different for each symbol $j$ to be transmitted to each user $i$.

We have obtained an expression for the power allocation that is still highly complex. However, this procedure gives us an insight on how to build alternative simplified schemes based on the same idea of the wellknown WF. Although suboptimal, they may perform close to the optimal solution, with the advantage of a much lower optimization burden.

\section{Waterfilling distributions for suboptimal power allocation schemes \\ Modified waterfilling}

By analyzing the set of constraints in either (11) or (12), the solution that we propose is to reduce the problem by considering an equivalent virtual BS (antenna) that would lead to a single constraint equation. The underlying idea is that, instead of all the BSs (antennas) giving a constraint on the powers $P_{i j}$ allocated to each stream $j$ of user $i$, we choose the BS (antenna) that needs more power to transmit this user information stream and, hence, will be the first to violate the constraint if we increase $P_{i j}$. Defining the new quantities

$$
\Omega_{i j}^{\mathrm{BS}}=\max _{k=1, \ldots, M}\left(\sum_{l=1}^{t}\left|w_{i j}^{k l}\right|^{2}\right) \quad \Omega_{i j}^{\mathrm{An}}=\max _{\substack{k=1, \ldots, M \\ l=1, \ldots, t}}\left(\left|w_{i j}^{k l}\right|^{2}\right)
$$

the simplified optimization problem reduces the constraints to just one, becoming

$$
\begin{aligned}
& \max \left\{\sum_{i=1}^{N} \alpha_{j} \sum_{j=1}^{r} \log _{2}\left(1+\lambda_{i j} P_{i j}\right)\right\} . \\
& \sum_{i=1}^{N} \sum_{j=1}^{r} P_{i j} \Omega_{i j}^{\mathrm{BS} / \mathrm{An}} \leq P_{\operatorname{maxBS} / \mathrm{An}}
\end{aligned}
$$

It should be noted that this new constraint is more restrictive than all the previous ones. Therefore, if we satisfy this restriction, then we also fulfill the restrictions in (11) or (12). Application of the Lagrange multiplier technique gives the new function:

$$
\sum_{i=1}^{N} \alpha_{i} \sum_{j=1}^{r} \log _{2}\left(1+\lambda_{i j} P_{i j}\right)-\mu\left(\sum_{i=1}^{N} \sum_{j=1}^{r} P_{i j} \Omega_{i j}^{\mathrm{BS} / \mathrm{An}}-P_{\operatorname{maxBS} / \mathrm{An}}\right)
$$

partial derivatives of which, with respect to the powers $P_{i j}$, give the set of equations:

$$
\frac{\alpha_{i}}{\ln (2)} \frac{\lambda_{i j}}{1+\lambda_{i j} P_{i j}}-\mu \Omega_{i j}^{\mathrm{BS} / \mathrm{An}}=0 .
$$

Hence,

$$
P_{i j}+\frac{1}{\lambda_{i j}}=\frac{\alpha_{i}}{\ln (2) \mu \Omega_{i j}^{B S / A n}} .
$$

Therefore, the problem is equivalent to finding the Lagrange multiplier (or constant $\left.K_{\mathrm{MWF}}=\frac{1}{\ln (2) \mu}\right)$ such that, for all the power levels $P_{i j}$, the following equation holds:

$$
P_{i j}=\left[K_{\mathrm{MWF}} \frac{\alpha_{i}}{\Omega_{i j}^{\mathrm{BS} / \mathrm{An}}}-\frac{1}{\lambda_{i j}}\right]^{+} i=1, \ldots, N \quad j=1, \ldots, r
$$

where $K_{\text {MWF }}$ must be found to fulfill the constraints (11) or (12). This corresponds again to a WF distribution with variable waterlevel. However here, and unlike the optimal solution in (20), in the variable waterlevel, we have decoupled the term containing the Lagrange multiplier $K_{\mathrm{MWF}}$ from $\Omega_{i j}^{\mathrm{BS} / \mathrm{An}}$ and $\alpha_{i}$. That is, the problem reduces to finding the only unknown value $K_{\mathrm{MWF}}$ in (26), while the variability in the waterlevel is confined to the known parameters $\Omega_{i j}^{\mathrm{BS} / \mathrm{An}}$ and $\alpha_{i}$. This can be solved with the same type of algorithms that solve standard WF [20].

\section{Waterfilling}

In order to further simplify the solution to the optimization problem, we may consider the fact that, in a practical realization, the values of $\Omega_{i j}^{\mathrm{BS} / \mathrm{An}}$ are close to each other for all $i, j$. Then, we assume them to be constant and include that constant into the waterlevel to simplify the solution in (26) giving

$$
P_{i j}=\left[K_{\mathrm{WF}} \alpha_{i}-\frac{1}{\lambda_{i j}}\right]^{+} \quad i=1, \ldots, N \quad j=1, \ldots, r
$$

where again $K_{\text {WF }}$ must be found to fulfill the constraints (11) or (12). This corresponds to a WF distribution with the waterlevel modified only by the user priorities. In particular, for equal priorities, $\alpha_{i}=1 / N$, which corresponds to a standard WF.

\section{Numerical results}

In this section we compare the performance of the proposed modified waterfilling (MWF) of (26), waterfilling 
(WF) of (27), and the optimum solution found by numerical convex optimization (CVX) [21]. For the sake of comparison, we also include the rates achieved when using the scaled WF (SWF) proposed in [10] for per base station constraints, the results of [7], also for per base station constraints, and a uniform power distribution (UP). In the case of UP, the power allocated to each user stream is the same and corresponds to the maximum value that fulfills either constraints (11) or (12).

In the following subsection, we analyze the achievable rates for each scheme in a simple scenario to understand how close they perform without the influence of the fading model. Then, in the subsequent subsections, we analyze a more realistic scenario with the effects of imperfect channel estimation and of the feed-back delay in a time-varying channel, which outdates the current channel with respect to the one used for precoding and power assignment.

\section{Achievable rates}

In a simple two-BS, two-user case $(M=N=2)$, we consider a simplified channel model where the matrix channel entries are independent identically distributed complex Gaussian random variables with zero-mean and unit variance. We set $P_{\operatorname{maxBS}}=1$ and $P_{\operatorname{maxAn}}=1 / t$. We find the boundary $B$ of the region of achievable rates for each proposed scheme as $B(\alpha)=\alpha R_{1}+(1-\alpha) R_{2}$, for $\alpha \in$ $[0,1]$, with $\left(R_{1}, R_{2}\right)$ being the pairs in the achievable region. Figures 2 and 3 show the regions of mean achievable rates, averaged over 1,000 channel realizations, with the per base station and per antenna power constraints, comparing the three different approaches with the uniform power allocation as a reference. SWF is also shown when the per base station constraints are used. Different values of the number of transmit and receive antennas are considered. It can be seen that the gap between the achievable rates obtained with WF and MWF and the optimal solution CVX is very narrow for the case of per base station constraints, while for the per antenna constraints, the difference between CVX and the waterfilling distributions becomes more noticeable. In both cases, these rates are considerably higher than what is achieved by UP. When the power is constrained per base station the performance of WF and SWF are the same; however, the use of MWF can give a small improvement. The fact that MWF performs better than WF is more visible when the power is constrained per antenna, because $\Omega_{i j}^{\mathrm{BS}}$ exhibit less variability and can be better approximated by a constant. In any case, the increase of mean achievable rates with higher values of $t$ and/or $r$ is substantial, meaning that the capabilities of multiple antennas are leveraged.
It is also interesting to analyze with more detail the behavior of UP in Figures 2 and 3 for $t=r=2$ and $t=2$, $r=1$. CBST is transmitting as many data streams per user as the number of receive antennas $r$ (10), each multiplied by the elements of the diagonal matrix $S_{i}\left(\lambda_{i j}^{1 / 2}\right)$ after the compound effect of transmit, channel, and receive processing. This means that one stream for $r=1$ and two streams for $r=2$ are transmitted using, therefore, 1 or 2 values of $\lambda_{i j}^{1 / 2}$ per user $i$. For each user, in these channel conditions, one of these values is generally considerably higher than the other, and so sharing the transmission power between two streams $(r=2)$ in the case of UP results in a waste of power that renders a lower rate than just using the entire available power in one stream $(r=1)$. One illustrative example from a particular channel realization: for $r=1$ we have $\lambda_{21}^{1 / 2}=1.4613, \lambda_{21}^{1 / 2}=1.4613$, while for $r=2$ we obtained $\lambda_{12}^{1 / 2}=0.6778$,

$\lambda_{22}^{1 / 2}=0.6878, \lambda_{21}^{1 / 2}=1.0839, \lambda_{22}^{1 / 2}=0.6878$. This is a well-known effect leading to the dominant eigenmode transmission concept described in [22].

Figure 4 shows the average achievable rates when users' transmissions have the same priority $(\alpha=0.5)$. Average rates (over 10,000 channel realizations) are plotted for different values of $t$ and $r$ and the two considered types of power restrictions. We can see that when the number of antennas is increased, the advantage of using the WF schemes over a uniform power distribution is more evident. The advantage of MWF over WF is relatively small, and they both perform close to CVX. Finally, we can observe that the per antenna power constraints, even though they may be more realistic, reduce the degrees of freedom in the power assignment, and therefore this leads to worse performance compared to the per base station power constraints. The effect is more noticeable for high antenna dimensions. In this figure, the mean achievable rates obtained with the JT-decomp precoding proposed in [7] for per base station power constraints are also shown. Since no optimization is performed on the transmit powers to maximize the achievable rates, the obtained mean rates are lower with this scheme.

\section{Effect of an erroneous or outdated channel estimation}

In the results of previous subsection, we assumed that the channel was perfectly estimated at each receiver and instantaneously fed back to the base stations so that BD insured that perfect cancelation of the interference was achieved. However, the channel is usually estimated at the receivers using the information conveyed by pilot symbols, and this estimation will normally be corrupted by additive white Gaussian noise (AWGN). Moreover, 


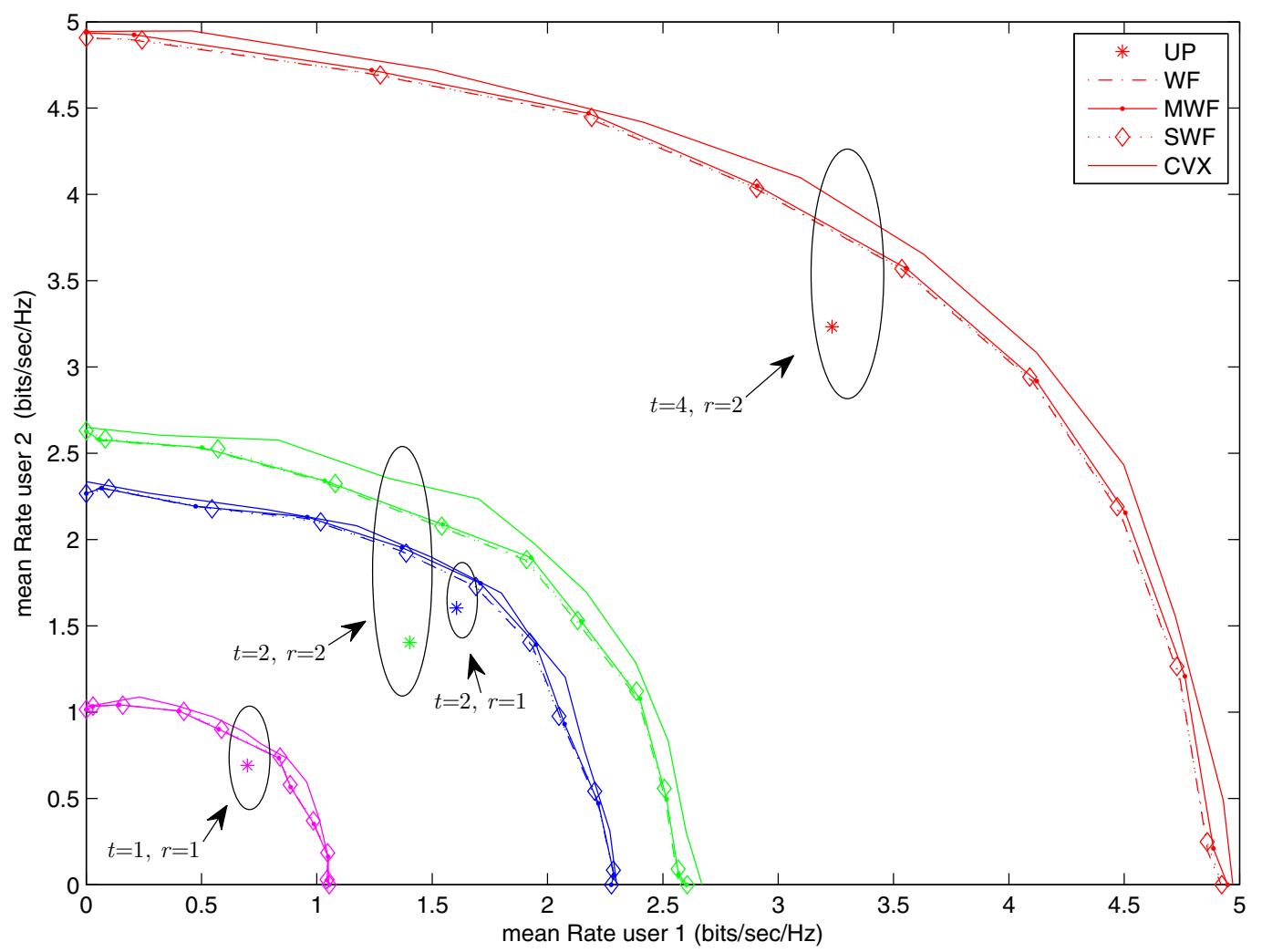

Figure 2 Mean achievable rates with per base station power constraints with $M=2, N=2$ and several values of the number of transmit $t$ and receive $r$ antennas.

sending the estimated channel state information (CSI) to the base stations will require some time, and therefore a delayed version of the estimated CSI will be available there. If $\mathrm{BD}$ is performed with erroneous or outdated CSI, then the diagonalization will not be perfect and some interference will remain. The power will be subsequently allocated using the wrong estimates. With the results shown in the subsequent figures, we discuss theses two effects.

The effects of imperfect channel estimation are evaluated using a noisy estimate of the channel matrix $\widehat{\mathbf{H}}$ instead of the real one

$$
\widehat{\mathbf{H}}=\mathbf{H}+\mathbf{H}_{\sigma}
$$

where $\mathbf{H}_{\sigma}$ is a matrix of i.i.d. complex Gaussian entries with zero mean and variance $\sigma_{H}^{2}$. BD is performed with the imperfectly estimated $\widehat{\mathbf{H}}$, and therefore the power allocation is determined using the singular values obtained with this estimation error. The mean squared error (MSE) of the channel estimation is defined as

$$
M S E=E\left[\frac{\|\mathbf{H}-\widehat{\mathbf{H}}\|_{F}^{2}}{\|\mathbf{H}\|_{F}^{2}}\right]=\sigma_{H}^{2}
$$

which is coincident with the normalized MSE of [23] where we can see [[23], Figure 4] that values of MSE lower than $10^{-1}$ can be achieved for operational numbers of antennas and signal-to-noise ratio (SNR) values.

The effects of the imperfect channel estimation are examined in Figure 5 for the case of per base station power constraints. If the mean rates that may be achieved with perfect channel estimation are denoted as $R_{\text {per }}$ and the mean rates achieved with imperfect channel estimation are denoted as $R_{\mathrm{imp}}$, then the relative loss $\left(R_{\text {per }}-R_{\text {imp }}\right) / R_{\text {per }}$ is plotted. We can observe that for reasonable values of the MSE obtained in the channel estimation (up to $10^{-1}$ ), the values of the relative loss are small, and so the degradation caused by imperfect channel estimation is not important. If the MSE increases above $10^{-1}$, then a degradation of the achievable rates can be observed, which increases with the number of 


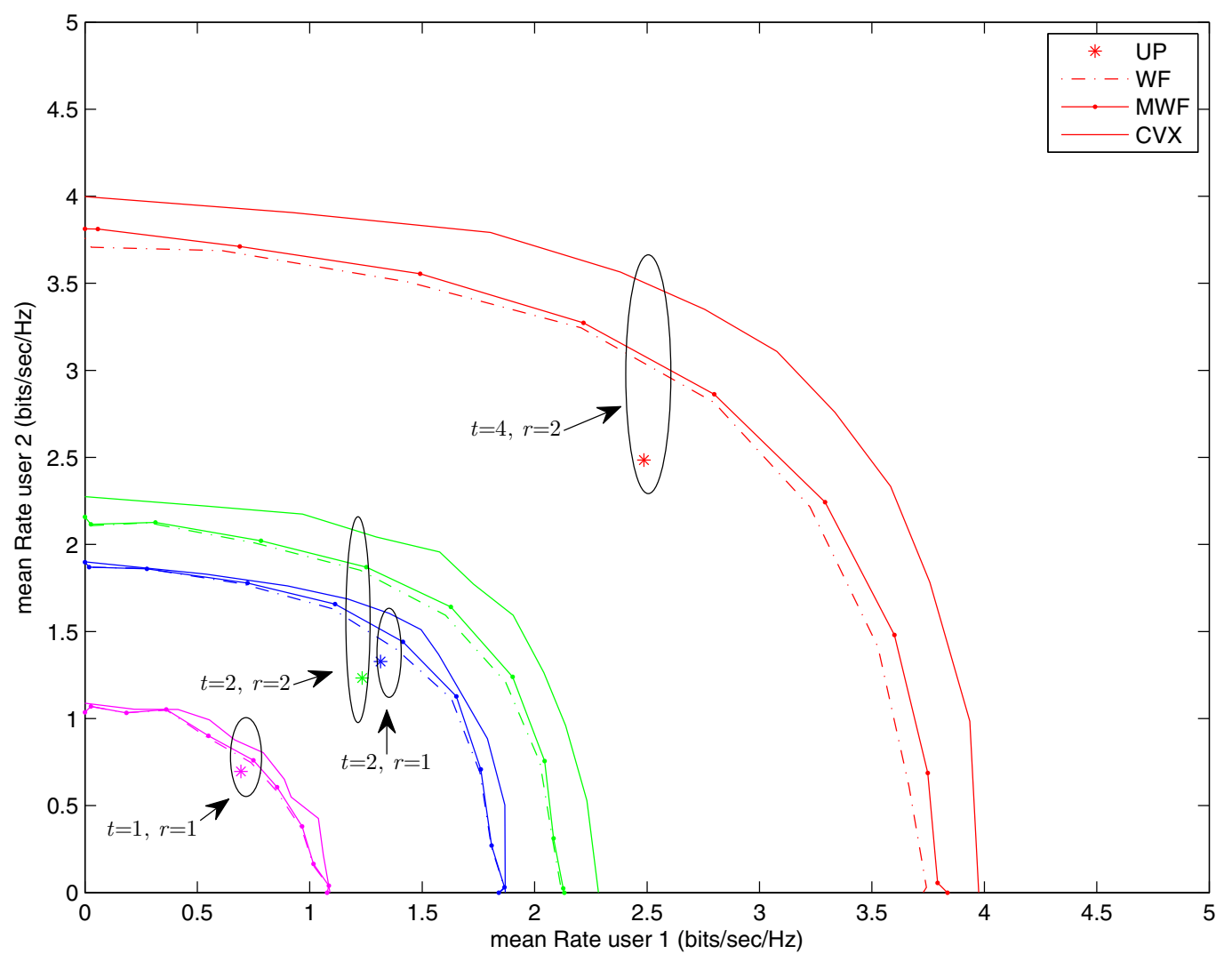

Figure 3 Mean achievable rates with per antenna power constraints with $M=2, N=2$ and several values of the number of transmit $t$ and receive $r$ antennas.

antennas. We can see that the degradation obtained when using WF and UP is in general quite similar. Although not included, the behavior of MWF is the same as WF.

The effects of outdated CSI are examined in Figures 6, 7 , and 8 for the case of per base station power constraints. Here, we have evaluated the performance in a more realistic scenario with $M=N=64$ as described in [6]. Cells of radius $d_{0}=1.6 \mathrm{~km}$ are arranged to form a torus which avoids the boundary effect that causes cells at the border of the cellular deployment to receive less interference. Each cell has a BS in its center and a single user allocated in the shared frequency, time-slot, or code resource of interest. The position of each user is randomly varied according to a uniform distribution over the area of each cell.

The channel-fading coefficients account for path loss with exponent decay 3.8, lognormal shadow fading with mean of $0 \mathrm{~dB}$ and standard deviation of $8 \mathrm{~dB}$, and Gaussian complex fading with zero mean, unit variance, and with a Doppler spectrum modeled by a Jakes filter [24] with maximum Doppler spread $f_{D}$. Given that in the system model we assume unit variance noise, we normalize the path loss $(P L)$ accordingly to account for different SNRs, which are specified at the cell boundary (at distance $d_{0}$ from the center) as

$$
S N R=\frac{P_{\operatorname{maxBS} / \mathrm{An}}}{P L\left(d_{0}\right)} .
$$

In this definition of SNRs, only the effect of path loss is included, not of the shadowing or fading, according to [6]. Also, it should be noted that the receivers placed closer to the BS will experience a higher value of SNR. Figures 6, 7, and 8 show the CDF of the rates achieved in this scenario when $t=r=1, t=r=2$, and $t=r=4$, respectively. The SNR at the cell boundary is $18 \mathrm{~dB}$. The parameter $D$ indicates the delay between the actual CSI and the CSI being used for the BD. That is, the CSI is outdated by a delay $D$ with respect to ideal CSI. In the figures, we show the performance for different 


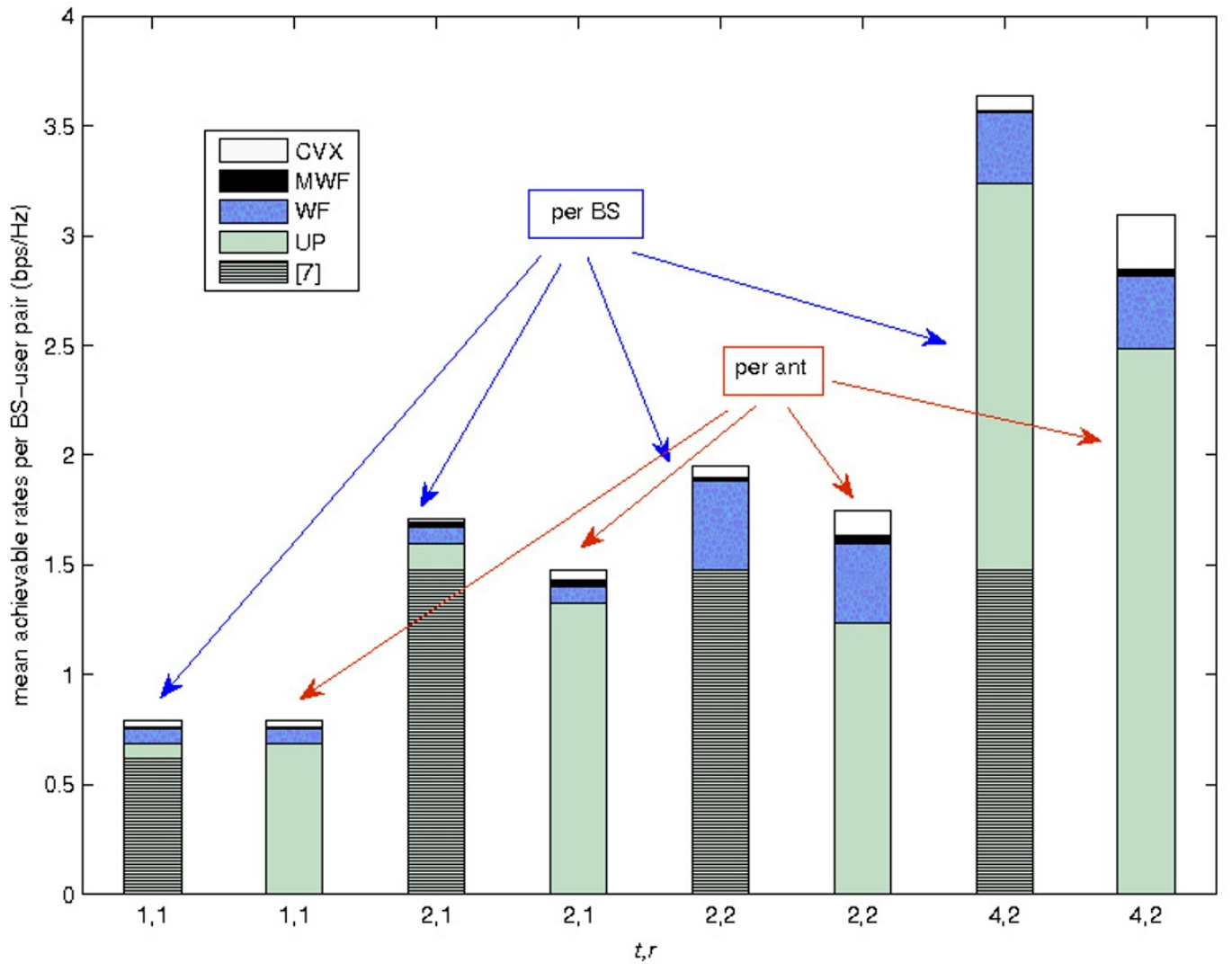

Figure 4 Mean achievable rates with $M=2, N=2$ and several values of the number of transmit $t$ and receive $r$ antennas.

values of $D$ conveniently normalized with respect to the channel coherence time $\left(T_{c}=1 / f_{D}\right)$. We can observe that the delay must be very small compared to the coherence time of the channel to cancel effectively the interference. When $D=0.001 T_{c}$, the degradation of the rates is already substantial. The degradation is more accentuated when transmitter and receiver have a higher number of antennas. Actually, with $D=0.005 T_{c}$, the advantages of increasing the number of antennas are lost, and the performance is basically the same for all the number of antennas considered in these figures.

A value $D=0.001 T_{c}$ is in line with the feed-back delay used in [25] to evaluate the performance of closed-loop MIMO systems $\left(D=0.1 \mathrm{~ms}\right.$ with $T_{c}=167 \mathrm{~ms}$, so $D=$ $0.0006 T_{c}$ ), while smaller values of delay seem infeasible in practice. Therefore, these results confirm that the use of outdated CSI can seriously degrade the performance of $\mathrm{BD}$, and therefore efficient feed-back mechanisms must be designed, which are beyond the scope of this article. We can note that UP and WF suffer approximately the same degradation (and also MWF not shown), while
CVX is more prone to the effects of the outdated CSI, which makes sense, since it strongly relays on the channel information to optimize the power allocation.

\section{Complexity}

The optimum power distribution can be obtained through a convex optimization procedure, while WF approaches allow a much reduced complexity at the expense of some performance degradation. In this section, we examine the difference in terms of complexity between both approaches for the power optimization procedure.

Since the power is distributed over $\mathrm{Nr}$ user transmissions, the complexity does not depend on the number of transmit antennas or base stations (as long as $M t \geq N r$ as required for $\mathrm{BD})$. Therefore, the complexity of WF, MWF, and CVX does not increase with the number of antennas per BS, which is a preferable characteristic, since often $t>$ $r$ in practice.

A thorough comparison of complexity of the methods is not easy, since the optimization procedures are adaptive with a number of operations that can vary according to 


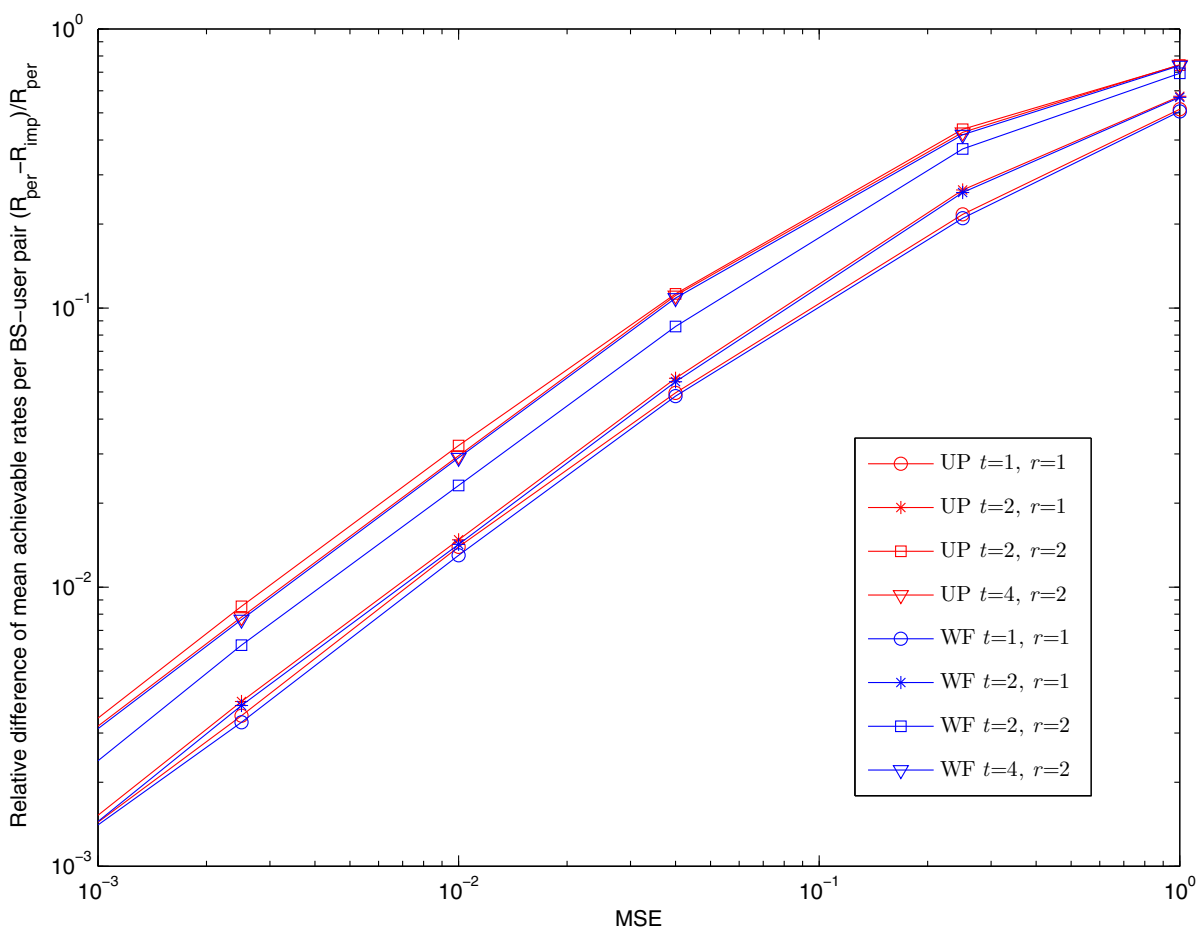

Figure 5 Ratio of mean achievable rates with $M=2, N=2$ as a function of the MSE of channel estimation.

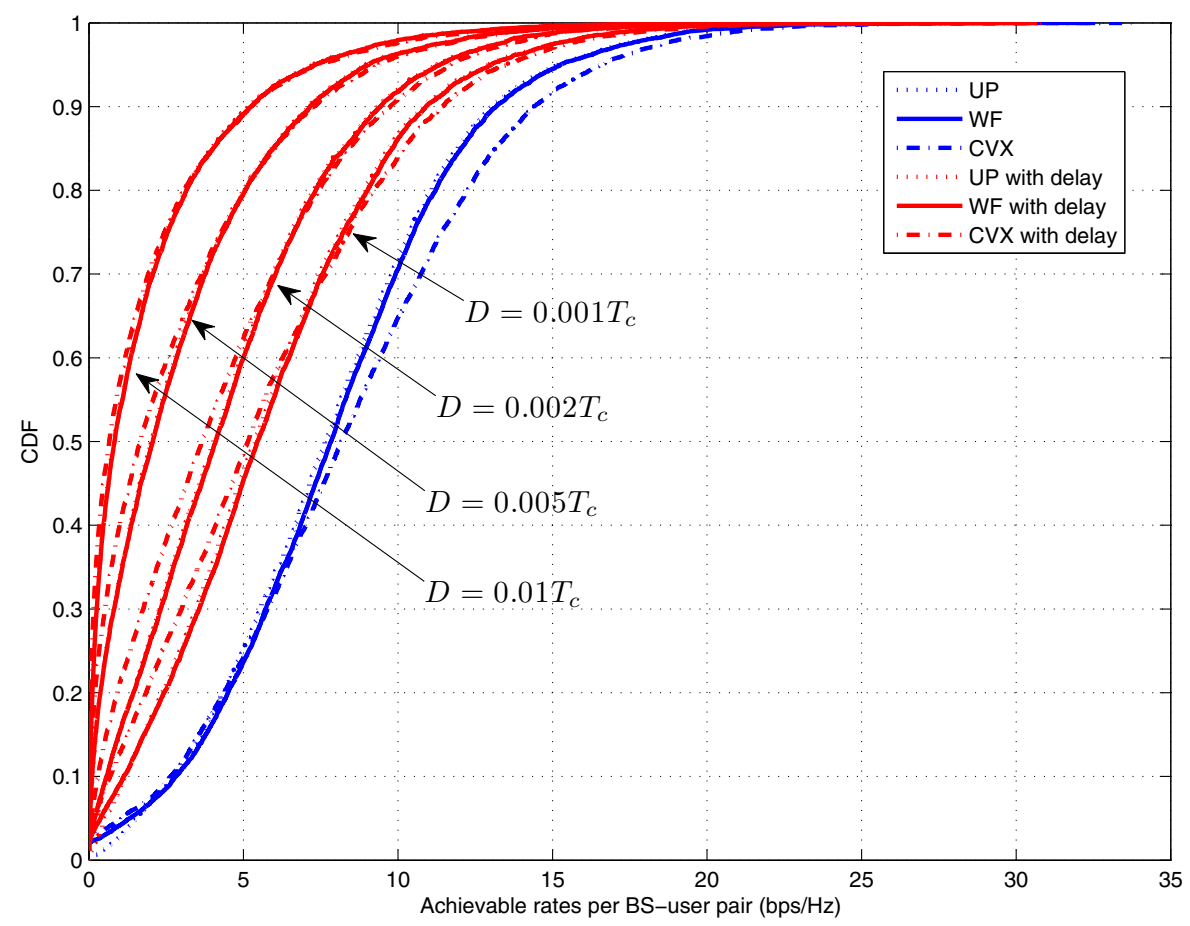

Figure 6 Bit rates obtained with $M=64, N=64, t=r=1$, per base station constraints, SNR $=18 \mathrm{~dB}$. 


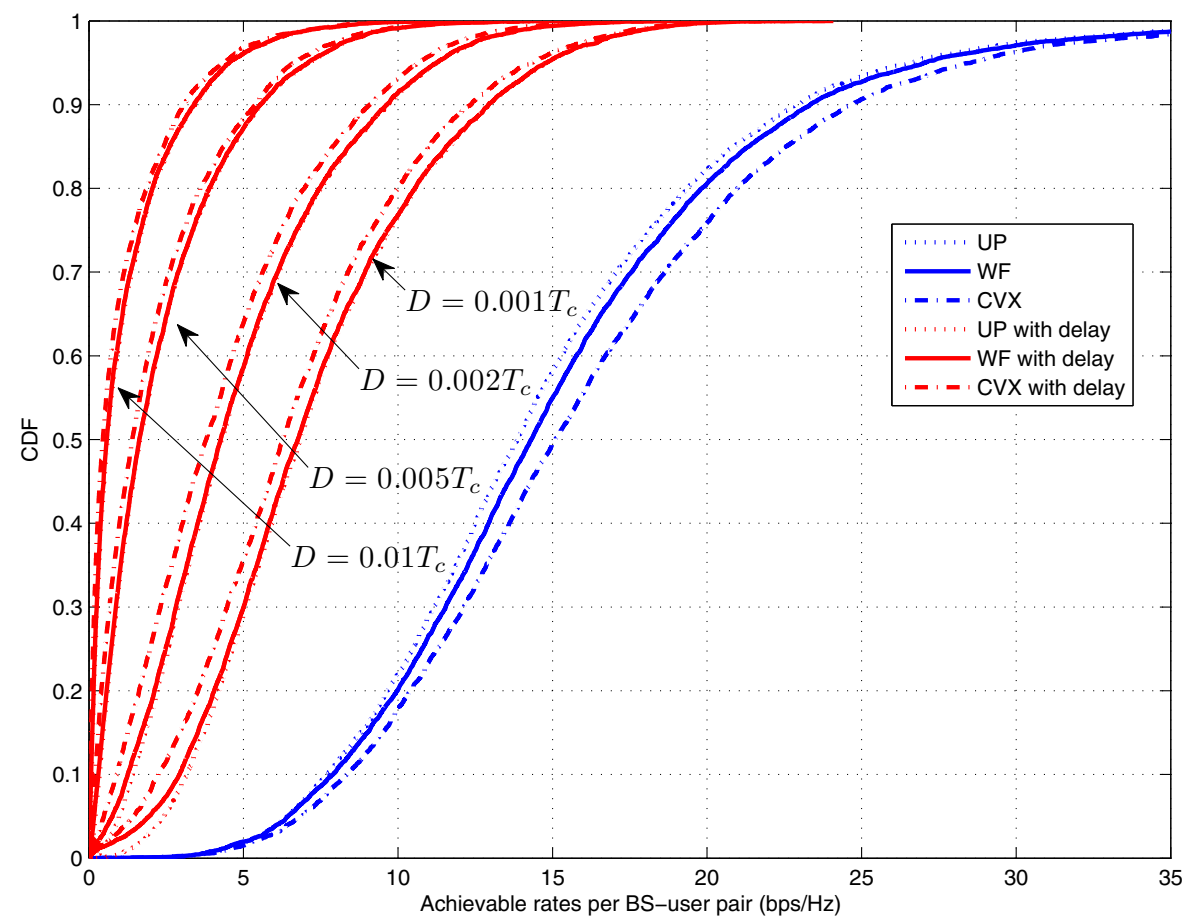

Figure 7 Bit rates obtained with $M=64, N=64, t=r=2$, per base station constraints, SNR $=18 \mathrm{~dB}$.

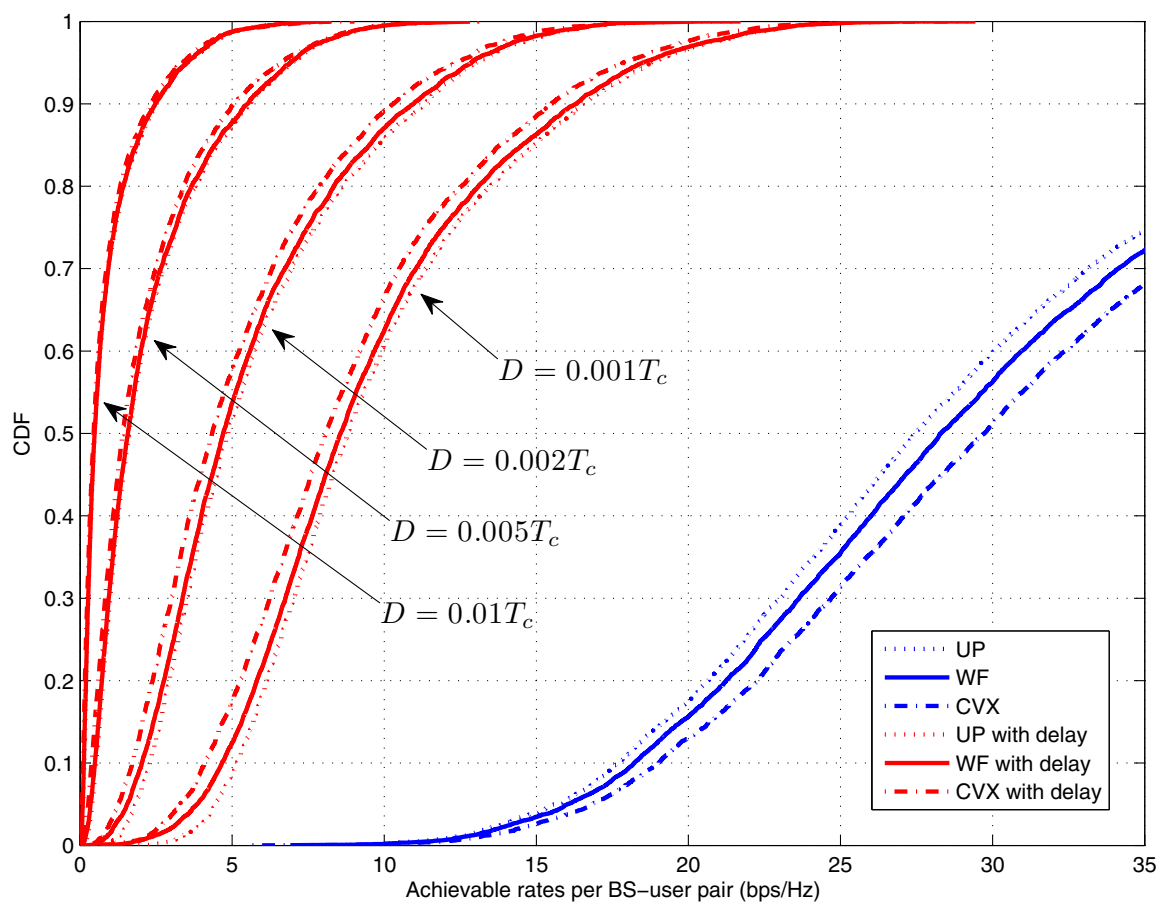

Figure 8 Bit rates obtained with $M=64, N=64, t=r=2$, per base station constraints, SNR $=18 \mathrm{~dB}$. 
the channel realization. In general, the convex optimization by using interior-point methods implements a Newton search with a number of iterations, which is slightly dependent on the problem size, and in most of the cases can be considered limited to few tens, while inside each Newton iteration, the complexity is dominated by the determination of the so-called Newton step which has a complexity order of about $(\mathrm{Nr})^{3} / 3$ [19]. For the WF, again, we can have a number of iterations variable with the channel conditions and the required accuracy; however, a theoretical number of operations for each iteration is on the order of $\mathrm{Nr} \log (\mathrm{Nr})$. In the specific case of modified WF, the search procedure cannot be optimized as in the WF, because of the variable waterlevel, and the complexity saving with respect to the convex optimization is lower.

To get a practical idea, we denote by $T_{\mathrm{CVX}}, T_{\mathrm{WF}}$, and $T_{\mathrm{MWF}}$ the mean execution time of CVX, WF, and MWF, respectively, all averaged over 1,000 channel realizations, and we plot in Figure 9 the ratios $T_{\mathrm{CVX}} / T_{\mathrm{WF}}$ and $T_{\mathrm{CVX}} /$ $T_{\text {MWF }}$ varying the number of cooperating base station- user pairs with per base station power constraints. For illustration purposes, we have set the number of antennas per base station to $t=4$; however, the results do not depend on the value of this parameter. The execution times obtained for $r=1,2$, and 4 have been averaged and plotted in this figure. The channel conditions are the same as in the "Achievable rates" section. The simulations were run on an Intel Core 2 Duo CPU at $2.53 \mathrm{GHz}$ with $2.00 \mathrm{~GB}$ RAM; however, since we are dealing with time ratios, the same values can be expected in other processors.

We have to keep in mind that the specific code implementation of the convex optimization and WF will have an impact on the measured times, and so what we give here is just an idea of their relative execution times. Having said that, we can observe that both WF and MWF are always more than three orders of magnitude faster than CVX. More specifically, WF is between 6,000 and 12,000 times faster, while MWF is between 1,000 and 2,000 times faster. Therefore, even if they are suboptimum, WF

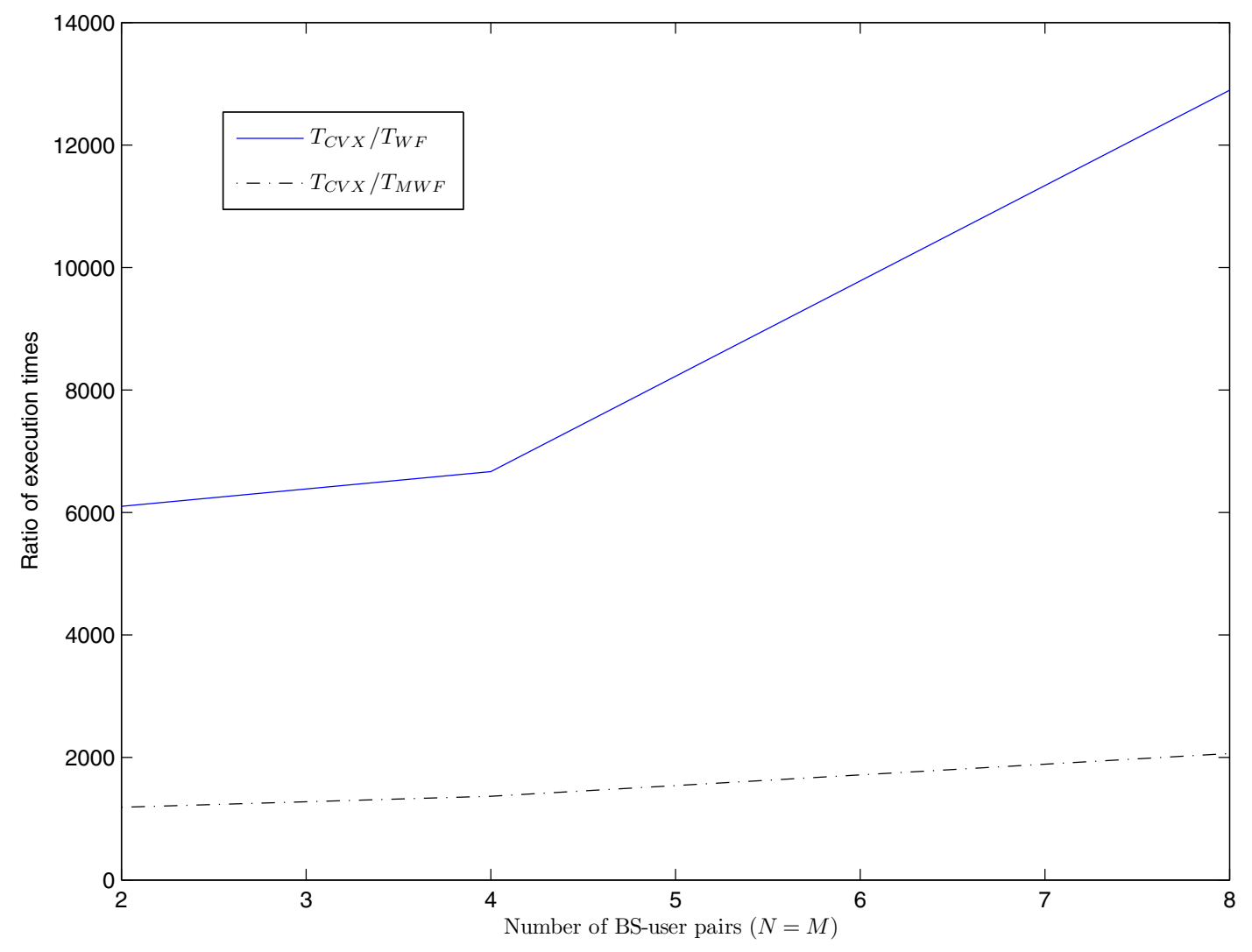

Figure 9 Ratio of the execution times of the different algorithms, averaged over $r=1,2$, and 4, for several values of $M=N, t=4$ and per base station constraints. 
and MWF make a good choice in terms of the balance between complexity and achievable rates.

\section{Conclusions}

We have proposed two power optimization schemes (WF and MWF) for the CBST downlink based on BD with different transmit power constraints. Both are derived with a technique similar to the WF distribution: the first (WF) has the lowest complexity and reduces to the standard WF if the user priorities are the same. In the case of per base station constraints, it achieves the same performance as SWF of [10]. The second (MWF) shows a better performance, more noticeable for the case of per antenna constraints, with a small increase in complexity. They both perform close to the optimal solution. However, the optimum can be derived only by resorting to the numerical solution of the convex optimization problem, with a heavy computational complexity, much higher than the proposed schemes of WF and MWF. Also, the degradation in terms of mean rates caused by imperfect channel estimation is small for reasonable values of the MSE of the channel estimation. However, our simulation results confirm the need of a fast feed-back of the estimated CSI to the base stations to avoid a severe degradation of the rates.

We have observed that the rates achieved with the more realistic per antenna constraints are lower compared to a per base station one. In general, the proposed schemes allow us to obtain the capacity improvements of MIMO, canceling the high amount of interference which characterizes cellular environments.

In [26], it is shown that, in the context of the Broadcast Channel, the performance of BD gets very close to DPC with a proper selection of the user scheduling. In further study, we will cope with the joint optimization of the power allocation, the precoding scheme, and the user scheduling.

\section{Endnotes}

${ }^{a}$ We assume, without loss of generality, that all the base stations have the same maximum available power.

${ }^{\mathrm{b}}$ Again, we assume, without loss of generality, that all the antennas in all the base stations have the same maximum available power.

\section{Acknowledgements}

This study has been partly funded by projects TEC2008-06327-C03-02, CCG10-UC3M/TIC-4620, and CSD2008-00010.

\footnotetext{
Author details

'Department of Signal Theory and Communications, Universidad Carlos III de Madrid, Leganés, Spain ${ }^{2}$ Department of Information Engineering, University of Padova, Padova, Italy
}

\section{Competing interests}

The authors declare that they have no competing interests.
Received: 25 March 2011 Accepted: 10 October 2011 Published: 10 October 2011

\section{References}

1. G Foschini, M Gans, On limits of wireless communications in a fading environment when using multiple antennas. Wireless Pers Commun. 6(3), 311-335 (1998). doi:10.1023/A:1008889222784

2. D Gesbert, S Kiani, A Gjendemsj, G len, Adaptation, coordination, and distributed resource allocation in interference-limited wireless networks. Proc IEEE. 95(12), 2393-2409 (2007)

3. S Shim, JS Kwak, JRW Heath, J Andrews, Block diagonalization for multi-user MIMO with other-cell interference. IEEE Trans Wireless Commun. 7(7), 2671-2681 (2008)

4. J Andrews, W Choi, R Heath, Overcoming interference in spatial multiplexing MIMO cellular networks. IEEE Wireless Commun. 14(6), 95-104 (2007)

5. S Shamai, B Zaidel, Enhancing the cellular downlink capacity via coprocessing at the transmitting end, in Proceedings of IEEE Vehicular Technology Conference (Rhodes, Greece) 1745-1749 (May 2001)

6. M Karakayali, G Foschini, R Valenzuela, Network coordination for spectrally efficient communications in cellular systems. IEEE Wireless Commun. 13(4) 56-61 (2006). doi:10.1109/MWC.2006.1678166

7. H Zhang, H Dai, Cochannel interference mitigation and cooperative processing in downlink multicell multiuser MIMO networks. EURASIP J Wireless Commun Netw. 2004(2), 222-235 (2004)

8. QH Spencer, AL Swindlehurst, M Haardt, Zero-forcing methods for downlink spatial multiplexing in multiuser MIMO channels. IEEE Trans Signal Process. 52(2), 461-471 (2004). doi:10.1109/TSP.2003.821107

9. BC Lim, W Krzymien, C Schlegel, Efficient sum rate maximization and resource allocation in block-diagonalized space-division multiplexing. IEEE Trans Vehicular Technol. 58, 478-484 (2009)

10. J Zhang, R Chen, J Andrews, A Ghosh, R Heath, Networked MIMO with clustered linear precoding. IEEE Trans Wireless Commun. 8(4), 1910-1921 (2009)

11. W Liu, S Ng, L Hanzo, Multicell cooperation based SVD assisted multi-user mimo transmission, in Proceedings of IEEE Vehicular Technology Conference (Barcelona, Spain) 1-5 (April 2009)

12. P Marsch, G Fettweis, On base station cooperation schemes for downlink network MIMO under a constrained Backhaul, in Proceedings of IEEE Global Communications Conference (Stoneham, Butterworth-Heinemann, New Orleans, USA, December 2008), pp. 1-6

13. $\mathrm{W} Y \mathrm{u}, \mathrm{T}$ Lan, Transmitter optimization for the multi-antenna downlink with per-antenna power constraints. IEEE Trans Signal Process. 55(6), 2646-2660 (2007)

14. RG Gallager, Information Theory and Reliable Communication (Wiley, New York, 1968)

15. RS Cheng, S Verdu, Gaussian multiaccess channels with ISI: capacity region and multiuser water-filling. IEEE Trans Inf Theory 39(3), 773-785 (1993). doi:10.1109/18.256487

16. W Yu, W Rhee, S Boyd, J Cioffi, Iterative water-filling for gaussian vector multiple access channels. IEEE Trans Inf Theory 50, 145-151 (2004). doi:10.1109/TIT.2003.821988

17. A García-Armada, M Sanchez-Fernández, R Corvaja, Waterfilling schemes for zero-forcing coordinated base station transmission, in Proceedings of IEEE Global Communications Conferences, Honolulu, USA, 1-5 (December 2009)

18. DP Palomar, Y Jiang, in MIMO Transceiver Design Via Majorization Theory (Foundations and Trends in Communication and Information Theory, MA, USA, 2006)

19. S Boyd, L Vandenberghe, Convex Optimization (Cambridge University Press, New York, 2004)

20. T Starr, J Cioffi, P Silverman, Understanding Digital Subscriber Line Technology, (Prentice Hall, New Jersey, 1999)

21. CVX: Matlab Software for Disciplined Convex Programming. http://cvxr.com/ cvx (June 2009)

22. F Boccardi, H Huang, A near-optimum technique using linear precoding for the MIMO broadcast channel, in Proceedings of IEEE International Conference on Acoustics, Speech and Signal Processing (Honolulu, USA, April 2007) pp. ||$|-17-|||-20$

23. H Zamiri-Jafarian, S Pasupathy, Robust and improved channel estimation algorithm for MIMO-OFDM systems. IEEE Trans Wireless Commun. 6(6), 2106-2113 (2007) 
24. WC Jakes, Microwave Mobile Communications (Wiley, New York, 1975)

25. SH Ting, K Sakaguchi, K Araki, A Markov-Kronecker model for analysis of closed-loop MIMO systems. IEEE Commun Lett. 10(8), 617-619 (2006). doi:10.1109/LCOMM.2006.1665129

26. T Yoo, A Goldsmith, On the optimality of multiantenna broadcast scheduling using zero-forcing beamforming. IEEE J Selected Areas Commun. 24(3), 528-541 (2006)

doi:10.1186/1687-1499-2011-125

Cite this article as: García Armada et al:: Constrained power allocation schemes for coordinated base station transmission using block diagonalization. EURASIP Journal on Wireless Communications and Networking 2011 2011:125.

\section{Submit your manuscript to a SpringerOpen ${ }^{\mathcal{O}}$ journal and benefit from:}

- Convenient online submission

- Rigorous peer review

- Immediate publication on acceptance

- Open access: articles freely available online

- High visibility within the field

- Retaining the copyright to your article

Submit your next manuscript at $\gg$ springeropen.com 